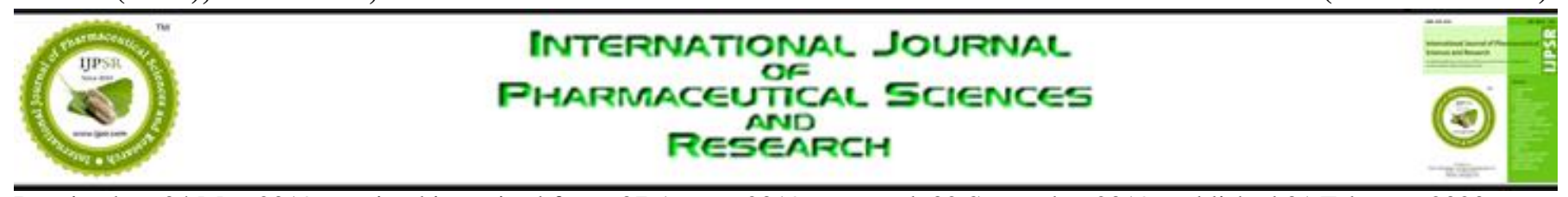

Received on 04 May 2019; received in revised form, 07 August 2019; accepted, 02 September 2019; published 01 February 2020

\title{
MICROSPONGES: AS A TOPICAL DRUG DELIVERY SYSTEM
}

\author{
Shailesh Sharma *, Anu Sharma and Chamanpreet Kaur
}

Department of Pharmaceutics, Pharmaceutical Research Division, Amar Shaheed Baba Ajit Singh Jujhar Singh Memorial College of Pharmacy, BELA (Ropar) - 140111, Punjab, India.

Keywords:

Microsponge,

Control drug release delivery system, Porous tiny microspheres

\section{Correspondence to Author:}

Dr. Shailesh Sharma

Professor,

Department of Pharmaceutics,

Pharmaceutical Research Division, Amar Shaheed Baba Ajit Singh Jujhar Singh Memorial College of Pharmacy, BELA (Ropar) - 140111, Punjab, India.

E-mail: shailesh.bela@gmail.com

\begin{abstract}
Now a day's control delivery of the drug is easily achieved by Microsponge delivery system. These microsponges are very similar to polymeric porous microspheres. In general microsponges are 5-300 micron in diameter, spherical in shape, large porous surface, tiny in structures. Microsponges generally used in the topical drug delivery system but recently they are used for oral administration of drugs. Microsponges deliver its drug on regular time intervals and also respond to the other stimulations. A microsponge has advantages that they modify the release of the drug enhances the stability and effectively reduces the side effects of the drugs. The main aim of formulation is to achieve desired concentration of the drug in the blood. When these microsponges used topically they prevent the accumulation of the drug in both dermis and epidermis. Microsponges can easily entrap and formulated into pharmaceutical product such as gels, creams, powders, liquids, and suspensions. One of the best features of microsponges is it has self-sterilization property; numerous study has confirmed that microsponges are non- mutagenic, nonirritant, non-allergic and non-toxic in nature. This article elaborates the history, advantages, preparation and evaluation and applications of microsponge.
\end{abstract}

INTRODUCTION: Drug delivery systems (DDS) that can precisely control the release rate or target drugs to a specific body site have had an enormous impact on the healthcare system. Carrier technology offers an intelligent approach for drug delivery by coupling the drug to a carrier particle (such as microspheres, nanoparticles, liposomes, etc.) which modulates the release and absorption characteristics of the drug ${ }^{1}$.

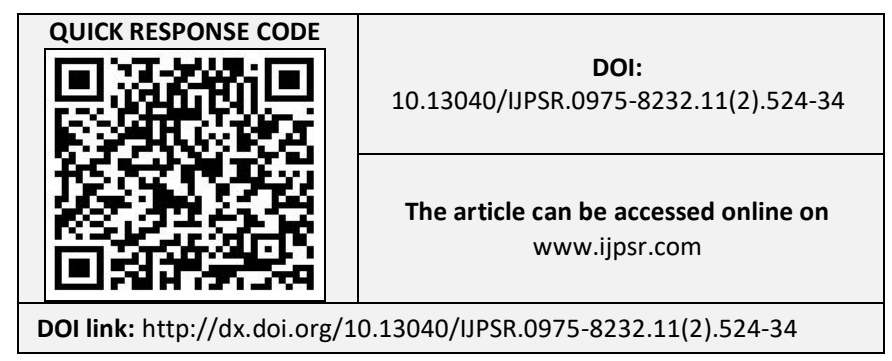

In recent years, there has been considerable emphasis given to the development of novel microsponge based drug delivery systems, to modify and control the release behavior of the drugs. By incorporation into a carrier system, it is possible to alter the therapeutic index and duration of the activity of drugs.

The ever-increasing interest among consumers with regard to skin care and skin treatment products has been fostered by the widespread use of ingredients like $\alpha$-hydroxy acids and vitamins in topical products, which can induce perceivable and demonstrable benefits like especially in aging or photodamaged skin. Although quite useful, in many instances, these ingredients may produce irritancy; such irritancy can be perceived as burning, stinging 
or redness and particularly occurs in individuals with sensitive skin. Recognizing this problem, the formulators have attempted to deal with this problem in one of the two methods. They have reduced the concentration of such ingredients, but in the process, sacrificed efficacy. They have also modified the vehicle in order to make the product more emollient or skin-compatible ${ }^{2}$. The major problem associated with TDS is most of the drugs are poorly water-soluble which pose many problems while formulating them in conventional dosage forms ${ }^{3}$. Another potential problem in topical drug delivery system of drugs-related is uncontrolled evaporation of the active ingredient, unpleasant odor, the use of unaesthetic vehicles which may be greasy, sticky, and may cause discolorations since this can result in the lack of patient compliance ${ }^{4,5,6}$.

Microsponge History: The microsponge technology was developed by Won in 1987, and the original patents were assigned to advanced polymer system, Inc. This company developed a large number of variations of the techniques and applied those to the cosmetic as well as OTC and physician prescribed products ${ }^{7}$.

Prospective Features of Microsponge: Microsponges are proved as a novel controlled pharmaceutical product. They can entrap a wide variety of drugs either in solid or in liquid form. They have high entrapment efficiency, they may absorb more than six times their weight. They are stable at high temperatures and $\mathrm{pH}$ ranging 1-11. They show good compatibility when they are suspended in other vehicles and liquid or semisolid dosage forms. They produce elegance of the product releases the drug in controlled manner, stable and nonirritating which improve the patient compliance ${ }^{8,9,10,11}$.

\section{Superiority over Other Pharmaceutical}

Formulations: Microsponge leads over-marketed pharmaceutical preparation.

A) Superior to Conventional Pharmaceutical Formulations: Generally semi-solid or biphasic liquid systems are available as topical delivery of drugs. They release the drug on the outer most layer of the skin. These conventional formulation showed rapid releases of the drug which may also absorb and accumulated in the epidermis and dermis layer of the skin. The high accumulation of the drug gives side effects, irritation, and toxicity. These problems are overcome with the microsponge drug delivery system. They release the drug gradually and in controlled manner to the skin ${ }^{12,13,14}$.

B) Over Micro and Nano-formulation: The pharmaceutical companies especially those companies who prepared controlled topical dosage forms shown their keen interest toward microsponge nowadays.

Microsponges are advantageous over the microsphere, microencapsulation, niosomes, lipid nanoparticles, nanotubes, liposomes etc. in the terms of their compatibility with large number of drugs (Ketoprofen, benzyl peroxide, retinol, fluconazole, ibuprofen, tertinoin, trolamine, prednisolone, acyclovir sodium, ticonazole) due to high capacity of drug loading, easy formulation technique, control release, physical, chemical and microbial stability $15,16,17,18,19,20,21$.

Formulation and Ingredient for Microsponge Preparation: Various polymers were reported to form microsponge 'cage'. Polymers studied for the fabrication of microsponges for the oral or topical purposes include Eudragit RS-100, Eudragit RS PO, Eudragit S-100, polylactide-co-glycolic acid, polylactic acid, polydivinyl benzene, and polyhydroxyl butyrate. Eudragit RS-100 formed the most widely studied polymer due to its versatility enabling the researchers to employ it in various ways. Eudragit RS PO also modulated the drug release along with enhancing the solubility of the drug by forming a solid dispersion like structure. Polylactide-co-glycolic acid and polylactic acid were studied for delivering the proteins and peptides.

Microsponges fabricated with these polymers also possessed floating ability due to the hydrophobicity of the polymer which limited the wetting of the particles with aqueous media, thus these microparticles can be employed for fabricating floating microsponges. Polydivinyl benzene was studied for fabricating the porous microparticles by polymerization technique using divinylbenzene as monomer but entrapping the drug with this process 
may cause alteration in structure of drug molecule or conjugation of drug with monomer. The use of such a large variety of polymers for the fabrication of the microsponges showed that the method of preparation of microsponges can be modified as per the requirement. In addition to polymers and active ingredients, some researchers also used triethyl citrate as plasticizer that helps to stabilize the resilient property of the microsponges. During the preparation of microsponges by quasi-emulsion solvent diffusion method, it is reported that the presence of an emulsifier having tendency to maintain the viscosity of aqueous phase is compulsory. Researchers attempted the use of cellulose ethers and PVA for such role and found the use of PVA as a better emulsifier ${ }^{22,23}$.

\section{List of Polymers used for the Preparation of Microsponge:}

- Ethylcellulose

- Eudragit RS 100

- Eudragit RL 100

- PHEMA

- Polystyrene

- Acrylic polymer

- Carbapol 934

- Polyvinyl alcohol

Preparation Methods of Microsponge: The drug loading in microsponge drug delivery system is done in two ways, the one-step process or by twostep processed as discussed in liquid-liquid suspension polymerization and Quasi emulsion solvent diffusion method which are based upon the physicochemical properties of the drug that is loaded to be. If the drug is typically inert non- polar material, will create the porous structure it is called porogen. Porogen drug, which neither hinders the polymerization nor becomes activated by it and stable to free radicals are entrapped with one step process $^{24}$.

\section{A) Liquid-Liquid Suspension Polymerization: In} general, a solution is made comprising of monomers and the functional or active ingredients, which are immiscible with water. This phase is then suspended with agitation in an aqueous phase, usually containing additives, such as surfactants and dispersants, to promote suspension. Once the suspension is established with discrete droplets of the desired size, polymerization is effected by activating the monomers either by catalysis, increased temperature or irradiation.

Polymerization process produced 1000 of microsponge cages which are spherical in structure interconnected with each other seems to look like a grapes bunch. On completion of polymerization produced solid particles were recovered from the suspension. Particles were washed and dried for further use ${ }^{25}$.

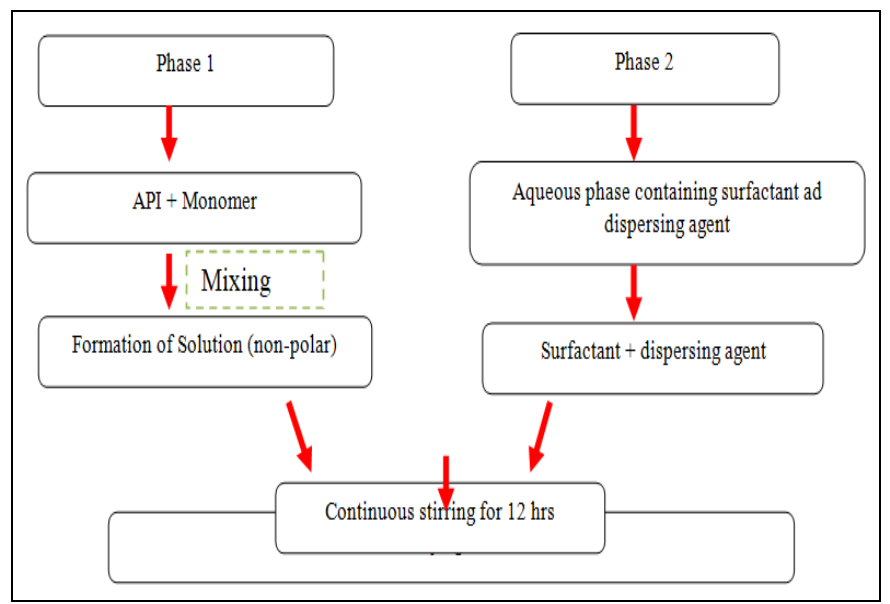

FIG. 1: PREPARATION OF SUSPENSION FOR LIQUID-LIQUID SUSPENSION POLYMERIZATION

B) Quasi-Emulsion Solvent Diffusion: This method is generally used for the preparation of oral and topical microsponges. In this method two phases were prepared, inner organic phase and outer aqueous phase.

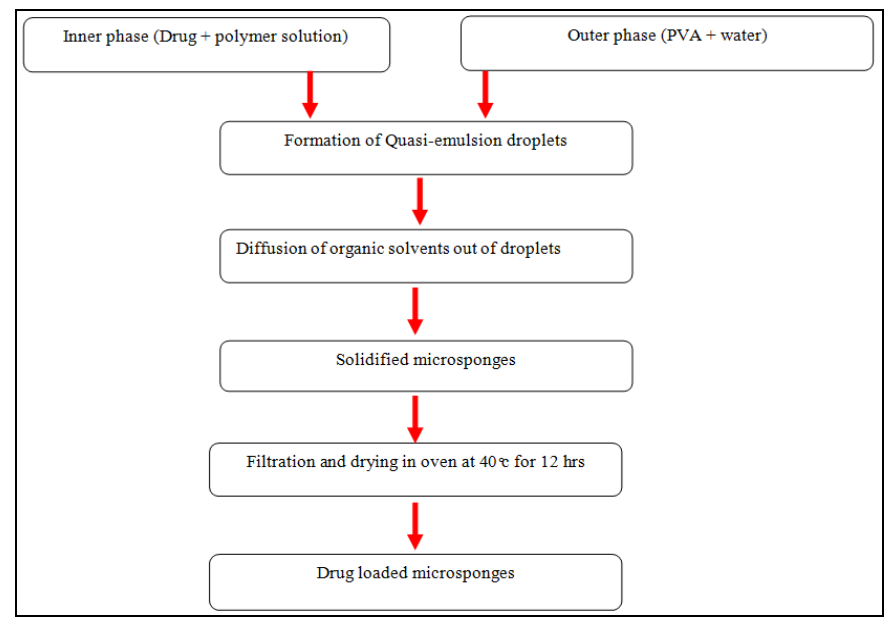

FIG. 2: QUASI-EMULSION SOLVENT DIFFUSION

In the inner organic phase polymer is dissolved in ethyl alcohol and drug is dissolved in this solution by ultrasonication at room temperature. The outer phase consists PVA solution in water. The solution 
is stirred and filtered for further use. The inner phase mixed in outer phase on mechanical stirrer dropwise. On the stirring the Quasi emulsion droplet was formed which may further evaporation of organic solvent produces the solid microsponge cages. The prepared microsponges are filtered and dried in oven for $12 \mathrm{~h}^{26,27,28}$.

C) Multiple-emulsion Solvent Diffusion: This novel technique was developed to prepare biodegradable porous microspheres. In this method, an internal aqueous phase containing an emulsifying agent like span, polyethyleneimine, and stearyl amine was dispersed in organic polymeric solution. Thereafter, this w/o emulsion was again dispersed in external aqueous phase containing PVA to form a double emulsion.

This method has the advantage of entrapping both water-soluble and water-insoluble drugs. It can also be used for entrapping thermolabile materials like proteins. Some authors also described the xanthan gum as an emulsifier to stabilize the internal w/o emulsion ${ }^{30,31}$.

D) Addition of Porogen: In this technique internal multiple emulsions was replaced by a porogen like hydrogen peroxide or sodium bicarbonate. For this, the porogen was dissolved in the polymeric solution to form a single-phase system which was redispersed in aqueous phase containing PVA. An initiator was then added to the multiple emulsion and the organic solvent was allowed to evaporate to leave the microparticles for producing microsponges ${ }^{32}$. The effect of incorporating hydrogen peroxide resulted in the formation of evenly distributed and interconnected pores with diameters ranging from 5 to $20 \mu \mathrm{m}$.

E) Oil in Oil Emulsion Solvent Diffusion: In contrast to w/o/w method, oil in oil (o/o) emulsion was prepared using volatile organic liquid as the internal phase that was allowed to evaporate slowly at a controlled rate with continuous stirring. As reported the technique used dichloromethane as the solvent for internal phase, polylactide glycolic acid as polymer and a mixture of fixed oil (corn or mineral) and dichloromethane containing span 85 as external phase. The internal phase was added dropwise to the dispersion medium with continuous stirring to get the microsponges. This technique was utilized for development of hydroxyzine $\mathrm{HCl}$ - loaded Eudragit RS-100 microsponges using acetone as dispersing solvent and liquid paraffin as the continuous medium. Selection of the organic solvent and external phase depend on the physicochemical properties of the drug and the polymer used for fabrication of microsponges ${ }^{33,34}$.

F) Lyophilization: Lyophilization as the technique was used for converting the microspheres prepared by gelation technique, to porous microspheres. In this methodology, the microspheres were incubated in the solution of chitosan hydrochloride and then lyophilized. Quick removal of solvent led to formation of pores in the microspheres. This method is quick and rapid but has the disadvantage of producing cracked or shrunken microparticles due to quick elimination of solvent ${ }^{35}$.

\section{G) Vibrating Orifice Aerosol Generator} Method: Vibrating orifice aerosol generator (VOAG) was first reported for the preparation of lipid bilayered mesoporous silica particles. The method involved the synthesis of porous particles by evaporation-driven surfactant templating in microdroplets by a VOAG method. For the preparation of core particle tetraethylorthosilicate, ethanol, water and dilute hydrochloric acid were refluxed to prepare stock solution. The stock solution was diluted with the solvent containing surfactant and stirred to allow the formation of monodisperse droplets using VOAG. The microspheres produced were encapsulated in the liposomes. These encapsulated particles can be utilized for targeted drug delivery of actives ${ }^{36}$.

H) Ultrasound-Assisted Production: This method was developed by modifying the liquid-liquid suspension polymerization. The microsponges are synthesis by utilizing the monomer betacyclodextrin (BCD) and cross-linking agent diphenyl carbonate. Size control of the microparticles was accomplished by heating and sonication of the reaction mixture. Then reaction mixture was allowed to cool, the product obtained was milled to give rough particles that were washed with distilled water and then by ethanol. The porous microparticles of cross-linked $\beta-C D$ can serve as carrier for efficient loading of drugs. However, this method has the limitation of entrapment of residues of the cross-linking agents that can be potentially toxic ${ }^{17}$. 
J) Electrohydrodynamic Atomization Method: Porous microsphere of chitosan was produced by this method. Chitosan solution was sonicated to generate bubbles and the resultant bubble suspension was drawn into a syringe, perfused through a steel capillary using a syringe pump and finally subjected to electrohydrodynamic atomization. The diameter of the capillary was chosen to retain all bubbles in the suspension while it flowed through it. The voltage used in the experiments solely depends on the concentration of chitosan in the solution. The combination of flow rate and applied voltage resulted in the stable conejet mode in each case, except when highest concentration was used that was difficult to electrospray. The chitosan microspheres were cross-linked by $4 \% \mathrm{w} / \mathrm{v}$ sodium hydroxide aqueous solution ${ }^{37}$.

Release Mechanism: The active ingredient entrapped in the microsponges may release by 4 mechanisms:

A) Pressure Triggered Release Mechanism: The entrapped drug is released from microsponge when they are pressurized or rubbed. The amount released depends upon the size and number of pore available on the sponge ${ }^{38}$.

B) Temperature triggered Release Mechanism: The active ingredients loaded in microsponges are viscous at storage temperature. On the application onto the skin by the means of rubbing or increase in temperature reduces the viscosity the active drug may flow out vigorously the skin. Sometimes by increasing the temperature of the skin may enhance the fluidity of drug. The release of the drug is easily modulated by changing the temperature ${ }^{39,40,41}$.

C) pH Triggered Release Mechanism: In this mechanism microsponge is coated with the $\mathrm{pH}$ dependent polymers. On the specific $\mathrm{pH}$ these polymers either swelled or leached out from the microsponges. After leaching of $\mathrm{pH}$-dependent polymer the drug released from the Microsponges. Coating of the microsponge increases the application of drug delivery to site-specific delivery.

D) Solubility Triggered Release Mechanism: When water-soluble drug loaded in microsponge it release only in presence of water. The rate of drug release from microsponge can be triggered by the amount of aqueous medium ${ }^{42}$.

Applications of Microsponges Drug delivery Systems: Microsponges are designed to deliver the pharmaceutically active ingredient efficiently at the minimum dose and also to enhance stability, reduce side effects and modify drug release. Microsponges are porous, polymeric microspheres that are used mostly for topical but recently used for oral administration.

A) Microsponge for Topical Delivery: The microsponge systems are based on microscopic, polymer-based microspheres that can bind, suspend or entrap a wide variety of substances and then be incorporated into a formulated product, such as a gel, cream, liquid or powder. A single microsponge is as tiny as a particle of talcum powder, measuring less than one-thousandth of an inch in diameter. Like a true sponge, each microsphere consists of a myriad of interconnecting voids within a noncollapsible structure that can accept a wide variety of substances. The outer surface is typically porous, allowing the controlled flow of substances into and out of the sphere.

Several primary characteristics, or parameters, of the microsponge system, can be defined during the production phase to obtain spheres that are tailored to specific product applications and vehicle compatibility. Microsponge systems are made of biologically inert polymers. Extensive safety studies have demonstrated that the polymers are non-irritating, non-mutagenic, non-allergenic, nontoxic and non-biodegradable. As a result, the human body cannot convert them into other substances or break them down. Although they are microscopic in size, these systems are too large to pass through the stratum corneum when incorporated into topical products ${ }^{35}$.

Benzoyl peroxide (BPO) is commonly used in topical formulations for the treatment of acne, with skin irritation as a common side effect. It has been shown that the controlled release of BPO from a delivery system to the skin could reduce the side effect while reducing percutaneous absorption. Therefore, microsponge delivery of benzoyl peroxide was developed using an emulsion solvent diffusion method by adding an organic internal 
phase containing benzoyl peroxide, ethylcellulose and dichloromethane into a stirred aqueous phase containing polyvinyl alcohol and by suspension polymerization of styrene and divinylbenzene. The prepared microsponges were dispersed in a gel base, and microsponge gels are evaluated for antibacterial and skin irritancy. The entrapped system released the drug at slower rate than the system containing free BPO. Topical delivery system with reduced irritancy was successfully developed ${ }^{43-53}$.

B) Microsponge for Oral Drug Delivery: In oral applications, the microsponge system has been shown to increase the rate of solubilization of poorly water-soluble drugs by entrapping such drugs in the microsponge system's pores. As these pores are very small, the drug is in effect reduced to microscopic particles and the significant increase in the surface area thus greatly increases the rate of solubilization.

Controlled oral delivery of ibuprofen microsponges is achieved with an acrylic polymer, eudragit RS, by changing their intraparticle density ${ }^{50}$. Sustained release formulation of chlorpheniramine maleate, using powder-coated microsponges, is prepared by the dry impact blending method, for oral drug delivery ${ }^{54}$. Controlled oral delivery of Ketoprofen prepared by quasi-emulsion solvent diffusion method with Eudragit RS 100 and afterward tablets of microsponges were prepared by the direct compression method. Results indicated that compressibility was much improved in the physical mixture of the drug and polymer; due to the plastic deformation of the sponge-like microsponge structure, producing mechanically strong tablet ${ }^{55}$.

C) Microsponge-based Delivery Systems for Bone and Tissue Engineering: Bone-substitute compounds were obtained by mixing prepolymerized powders of polymethylmethacrylate and liquid methylmethacrylate monomer with two aqueous dispersions of a-tricalcium phosphate grains and calcium-deficient hydroxyapatite powders. The final composites appeared to be porous and acted as microsponges ${ }^{56}$. The basic fibroblast growth factor incorporated in a collagen sponge sheet was sustained release in the mouse sub-cutis according to the biodegradation of the sponge matrix and exhibited local angiogenic activity in a dose-dependent manner. Intramuscular injection of collagen microsponges incorporating fibroblast growth factor, induced a significant increase in the blood flow, in the murine ischemic hind limb, which could never have been attained by the bolus injection of $\mathrm{T}$ fibroblast growth factor these results suggest the significance and therapeutic utility of the type I collagen as a reservoir of fibroblast growth factor ${ }^{57}$.

A biodegradable graft material containing the collagen microsponge was developed for cardiovascular tissue grafting, as it would permit the regeneration of the autologous vessel tissue ${ }^{59}$. A thin biodegradable hybrid mesh of synthetic poly (DL-lactic-co-glycolic acid) (PLGA) and naturally derived collagen was used for three-dimensional culture of human skin fibroblasts. The hybrid mesh was constructed by forming web-like collagen microsponges in the openings of a PLGA-knitted mesh ${ }^{61}$. A tissue-engineered patch made of our biodegradable polymer and collagen-microsponge provided well in situ regeneration at both the venous and arterial wall, suggesting that this patch could be used as a novel surgical material for the repair of the cardiovascular system ${ }^{60}$.

Evaluation Methodology of Microsponge: ${ }^{61-74}$ Various evaluation tests were performed to evaluate the prepared formulations of microsponges:

\section{A) Total Yield Percentage and Loading} Efficiency: The total yield percentage of prepared Microsponge is calculated by the formula:

$\%$ Yield $=$ Actual weight of product/total weight of product $\times 100$

B) Scanning Electron Morphology (SEM): SEM by using the SEM the morphology surface topography and particle size diameter can be easily studied.

C) Characterization of Pore Structure: Pore volume and diameter are vital in controlling the intensity and duration of effectiveness of the active ingredient. Pore diameter also affects the migration of active ingredients from microsponges into the vehicle in which the material is dispersed. Mercury intrusion porosimetry can be employed to study effect of pore diameter and volume with the rate of drug release from microsponges. 
Porosity parameters of microsponges such as intrusion- extrusion isotherms pore size distribution, total pore surface area, average pore diameters, interstitial void volume percent porosity, percent porosity filled, shape and morphology of the pores, bulk and apparent density can be determined by using mercury intrusion porosimetry.

D) In-vitro Dissolution Studies: The dissolution pattern of the active ingredient of the microsponge can be estimated by USP dissolution apparatus. 900 $\mathrm{ml}$ of stimulated solution at $37 \pm 0.5^{\circ} \mathrm{C}$ are used to determine to dissolution behavior of the drug.

E) Stability Studies: The accelerated stability studies are carried out according to guidelines given by the International Council of Harmonization (ICH guidelines). The formulations are tested for stability at $50 \pm 2{ }^{\circ} \mathrm{C}, 250 \pm 2{ }^{\circ} \mathrm{C} / 60 \pm$ $5 \mathrm{RH}, 400 \pm 2{ }^{\circ} \mathrm{C} / 75 \pm 5 \mathrm{RH}$. Formulations are stored in glass bottles/vials and are evaluated after every $15,30,45$ days for their physiochemical characteristics.

F) Drug-Polymer Compatibility Studies: The sample of drug, excipients, and mixture of drug with excipients (binary (1:1) powder mixtures prepared by triturating drug with the individual excipients) was sealed in vials and kept at room temperature for not less than one month and then samples were analyzed by DSC, XRD, and FTIR.

Advancement in Microsponge Drug Delivery System: Pharmaceutical companies step ahead in the Microsponge technologies. Nowadays by modifying the method they are involved in nanosponges, nanoferrosponges, and porous microbeads. These formulations are more established and stable than the microsponges.

\section{A) Nanosponges: Nanosponges are the} nanoformulations which are used in topical drug delivery, especially the cosmetic agents passive targeting. These are helpful for absorption through the skin and extended retention in the layer of skin. These nanosponges developed by modifying the Solvent diffusion method. Either change in the agitation, amount of polymer and emulsifying agent can be easily produced nanosponges. Some researcher also showed that nanosponges are good carrier for the delivery of active ingredient which is available in gaseous form. These nanosponges carriers are also responsible for targeting cancerous cells $^{75-85}$.

B) Nanoferrospong: The nanoferrosponges are nano targeting devices consisting of ferric ions which may triggered with the help of magnet. The magnet enforces the carrier to stimulate to the deeper tissues and provide the drug at specific target site. These nanoferrosponges were prepared by co- precipitating of magnetic material with polymers. The prepared Nanoferrosponge have in high swelling index, excellent elasticity, hydrophilicity, and response to magnetism ${ }^{86-90}$.

C) Porous Microbeads: Improved characteristics of porous microsphere produces the microbeads which have a large number of pores. Polymerization and cross-linking technologies are used to developed solid porous microbeads. These microbeads are used for topical, buccal and oral drug delivery system ${ }^{91-92}$.

\section{TABLE 1: LIST OF MARKETED PRODUCTS BASED ON MICROSPONGES}

\begin{tabular}{ccc}
\hline Product Name & Pharmaceutical uses & Manufacturer \\
\hline Retinol cream & Helps maintain healthy skin & Biomedic \\
Salicylic Peel 20 & Excellent exfoliation & Biophor \\
Ultra Guard & Protects baby's skin & Scott Paper Company \\
Oil-free matte block SPF 20 & Sunscreen & Dermalogica \\
Benzoyl peroxide & Anti Acne & \\
Retin A Micro & Acne vulgaris & Ortho-McNeil-Pharmaceutical, Inc \\
Glycolic Acid Moisturizer w/SPF 15, & Anti-Wrinkles & AMCOL Health \& Beauty Solution \\
EpiQuin Micro & Hyper pigmentation & SkinMedicaInc \\
\hline
\end{tabular}

Future Aspects of Microsponge Drug Delivery System: Microsponges are one of the novel drug delivery systems, which were originally developed for topical delivery of drugs. They can also be used for tissue engineering and controlled oral delivery of drugs using biodegradable polymers. It provides a wide range of formulating advantages. Liquids can be transformed into free-flowing powders. 
Formulations can be developed with otherwise incompatible ingredients, with prolonged stability, without the use of preservatives. Therefore, microsponges will be an ideal drug delivery system related to formulations like the transdermal delivery system. As it requires vehicles at a higher concentration in order to dissolve the API for effective therapy, it causes irritation and hypersensitivity reactions in significant users.

Another demerit of topical formulations is uncontrolled evaporation of the active ingredient, unpleasant odor, and the potential incompatibility of drugs with the vehicles. Conventional formulations of topical drugs are intended to work on the outer layers of the skin. Typically, such products release their active ingredients upon application, producing a highly concentrated layer of an active ingredient that is rapidly absorbed. Thus, the need exists for a system to maximize the amount of time that an active ingredient is present either on the skin surface or within the epidermis. Some microsponge-based products are already approved; several others are currently under development and clinical assessment.

CONCLUSION: The microsponge delivery system is a unique technology for the controlled release of macroporous beads, loaded with an active agent, offering a potential reduction in side effects while maintaining their therapeutic efficacy. The microsponge drug delivery system offers entrapment of its ingredients and is believed to contribute toward reduced side effects, improved stability, increased elegance and enhanced formulation flexibility. In addition, numerous studies have confirmed that microsponge systems are non-irritating, non-mutagenic, non-allergenic, and non-toxic. This technology is being used currently in cosmetics, over-the-counter skincare, sunscreens, and prescription products. This kind of drug delivery technology may lead to a better understanding of the healing of several diseases. Hence, Microsponge-based drug delivery technology is likely to become a valuable drug delivery matrix substance for various therapeutic applications in the future.

ACKNOWLEDGEMENT: We acknowledge the support of the Sub-Committee, Pharmacy College and faculty members of COP- BELA.
CONFLICTS OF INTEREST: The author declared no conflicts of interest.

\section{REFERENCES:}

1. Bansode AS, Kute VB and Kote PS: Formulation, development and evaluation of microsponges loaded topical gel of nystatin. Journal of Drug Delivery and Therapeutics 2019; 9(2-s): 451-61.

2. Rajeshwari S, Swapna V: Microsponges as a neoteric cornucopia for drug delivery system. International Journal of Current Pharmaceutical Research 2019; 11(3).

3. Balamurugan $\mathrm{K}$, Kshirasagar $\mathrm{N}$ and Govardhan $\mathrm{P}$ : Microsponges: As a drug delivery system. The Pharmaceutical Innovation Journal 2019; 8(1): 139-43.

4. Shah CN and Shah DP: design and optimization of Fluconazole microsponges containing ethylcellulose for topical delivery system using quality by design approach. An International Journal of Pharmaceutical Science 2014; $5(3)$.

5. Osborne OW and Amann AH: Topical Drug Delivery Formulation. Marcel Dekker Publisher Inc: New York and Basel 1990: 308-309.

6. Kapoor D, Patel M and Vyas RB: A review non microsponge drug delivery system. Journal of Drug Delivery And Therapeutics 2014; 4(5): 29-35.

7. Pawar AP, Gholap AP, Kuchekar AB, Bothiraja C and Mali AJ: Formulation and evaluation of optimized oxybenzone microsponge gel for topical delivery. Journal of Drug Delivery 2015; 15: 261-68.

8. Patil SS, Kale A and Dandekar V: Microsponge drug delivery system: An overview. European Journal of Pharmaceutical and Medical Research 2016; 3(8): 212-21.

9. Bothiraja C, Gholap AD, Shaikh KS and Pawar AP: Investigation of ethylcellulose microsponge gel for topical delivery of eberconazole nitrate for fungal therapy. Journal of Therapeutic Delivery 2014; 5: 781-94.

10. Yadav V, Yadav $\mathrm{P}$ and Dombe $\mathrm{S}$ : Formulation and evaluation of microsponge gel for topical delivery of antifungal gel. International Journal of Applied Pharmaceutics 2017; (94).

11. Dineshmohan S and Gupta VRM: Formulation of fluconazole as topical antifungal gel by microsponge based delivery system. Indonesin Journal Pharmaceutical 2017; 28(3): 158-67.

12. Patel UB, Shah $\mathrm{CN}$ and Patel HM: Formulation and development of aceclofenac loaded microsponge for topical delivery using quality by design approach. International Journal of Advanced Pharmaceutics 2018; 7(4): 17-32.

13. Ahmad A, Makram M: An overview of microsponge as a novel tool in drug delivery. Modren Approaching in Drug Designing 2018.

14. Sonali and Rahul: Formulation and evaluation of prednisolone loaded microsponges for colon drug delivery: In-vitro and pharmacokinetic study. International Journal of Pharmaceutical Sciences and Research 2014; 5(5): 1994-05.

15. Verma P, Dhynai A and Juyal D: A brief review on microsponge use in chronopharmacology. The Pharmaceutical Innovation Journal 2018, 7(6): 538-43.

16. Jyoti and Kumar S: Innovative and novel strategy: Microsponge for topical drug delivery. Journal of Drug Delivery and Therapeutics 2018; 8(5): 28-34. 
17. Jagtap SC, Karale AA: Microsponge a novel topical drug delivery system. Journal of Drug Delivery Research 2014; 3(4).

18. Tile MK and Pawar A: Microspong: A novel strategy for drug delivery. International Journal of Pure and Applied Bioscience 2015; 3(1): 224-35.

19. Gupta NB, Sumbria R and Kumar K: Microsponges: topical preparations and its applications. World Journal of Pharmacy and Pharmaceutical Sciences 2017; 6(4): 62942.

20. Kumari P and Mishra SK: A comprehensive review on novel microsponge drug delivery approach. Asian Journal of Pharmaceutical and Clinical Research 2016; 9(1).

21. Makwana R, Patel H and Patel V: Microsponge for drug delivery system. International Journal of Pharmacy and Technology 2014; 5(4): 2839-51.

22. Shrivastva S, Kumar D and Singh PS: A review: Microsponge- An effective drug delivery system. Asian Journal of Pharmaceutical Research and Development 2017; 5(2): 1-8.

23. Kar AK, Kar B and Parya H: A novel approach on microsponge: Multifunctional modern dosage form International Journal Pharmaceutical Sciences' Review and Research 2018; 51(2): 64-72.

24. Sagavkar SR and Mohit SK: Innovative and novel strategy: Microsponges drug delivery system. International Journal of Universal Pharmacy and Biosciences 2014; 3(4).

25. Rajurkar VG, Tambe AB and Deshmukh VK: Topical anti-inflammatory gels of naproxen entrapped in eudragit based microsponge delivery system. Journal of Advanced Chemical Engineering 2015; 5(2): 2-6.

26. Nawal A and Mohammad S: Formulation and in-vitro evaluation of piroxicam microsponge as a tablet. International Journal of Pharmacy and Pharmaceutical Sciences, 2016; 8(2): 104-11.

27. Shukla A, Garg A and Garg S: Application of microsponge technique in drug delivery system. Asian Journal of Biomaterial Research 2016; 2(4): 120-26.

28. Mohit PB and Khanage SG: Recent advance in microsponge drug delivery system. Journal of Critical Review 2016; 3(1): 9-16.

29. Patel S, Seth A and Shah N: Microspong: A novel approach in gastro retention drug delivery system. Indo American Journal Pharmaceutical Research 2016; 6(7): 2231-76.

30. Bisht PS, Chopra $\mathrm{H}$ and Mishra S: Microsponge technology for novel topical drug delivery and oral drug delivery system: an overview. American Journal of Pharmatech Research 2016; 6(3): 2249-87.

31. Sruti TP, Thomos N and Daisy PA: Microsponge drug delivery system for topical delivery- A review. International Journal of Pharmaceutical, Chemical and Biological Science 2016; 6(4): 424-31.

32. Solanli D, Patidar S and Motiwale M: An overview of microsponge as a novel tool in drug delivery. International Journal of Pharmacy and Pharmaceutical Research 2017; 9(2): 2349-03.

33. Roy A: Microsponge as a novel drug carrier system: A review. World Journal of Pharmaceutical Research 2015; 4(12): 680-01.

34. Mantry S, Das S and Das S: Microsponge as novel strategy of drug delivery system. Universal Journal of Pharmaceutical Science and Research 2015; 1(1): 32-38.

35. Manda R and Naresh G: A review: Microsponge a novel new drug delivery system. Journal of Scientific Research in Pharmacy 2015; 4(1): 1-5.
36. Charde MS, Ghanawat PB, Welankiwar AS, Kumar J and Chakole RD: Microsponge a novel new drug delivery system: A review. International Journal of Advances in Pharmaceutics 2013; 2(6).

37. Vikrant K, Dolas RT, Somwanshi SB, Gaware VM, Kotade KB, Dhamak KB, Khadse AN and Kashid VA: Microparticles: a novel approach to enhance the drug delivery: A review. International $\mathrm{J}$ of Pharmaceutical Research and Development 2011; 3(8): 170-83.

38. Shah CN and Shah DP: Microsponges: A revolutionary path breaking modified drug delivery of topical drugs. International Journal of Pharmaceutical Research 2014; 6(2): 2-11

39. Bamane GS, Kakade TB and Kulkarni LV: Microsponges: A novel drug delivery system. World Journal of Pharmacy and Pharmaceutical Science 2014; 3(3): 748-62.

40. Pentewar RA, Kazi S and Bharti R: MDS Technology: An approach for topical, oral controlled and cosmetic formulations. Research Journal of Pharmaceutical, Biological and Chemical Sciences 2014; 5(3): 1170-90.

41. Deshwal S and Saxena AK: Microsponge delivery system: An overview. International Journal of Bio-pharmaceutics 2014; 5(1): 39-46.

42. Jelvehgari M, Siahi-Shadbad MR, Azarmi S, Gary P and Nokhodchi A: The microsponge delivery system of benzoyl peroxide: Preparation, characterization and release studies. International J of Pharmaceu 2006; 308: 124-132.

43. Draize JH, Woodard G and Calvery HO: Methods for the study of irritation and toxicity of substance as applied topically to the skin and mucous membranes. Journal of Pharmacology Experimental Therapeutics 1944; 82: 377 89.

44. D"souza JI: The Microsponge drug delivery system: For delivering an active ingredient by controlled time release. Pharma Info Net 2008; 6(3): 62.

45. Sarat PM, Ajay M, Nagendra BB, Prathyusha $P$, Audinarayana $\mathrm{N}$ and Bhaskar RK: microsponge drug delivery system: A review. Journal of Pharmaceutical Research 2011; 4(5): 1381-84.

46. D"souza JI: In-vitro antibacterial and skin irritation studies of microsponges of benzoyl peroxide. Indian Drugs 2001; 38(7): 23.

47. Wester R, Patel R, Natch S, Leyden J, Melendres J and Maibach $\mathrm{H}$ : Controlled release of benzoyl peroxide from a porous microsphere polymeric system can reduce topical irritancy. Journal of American Academy Dermatology 1991; 24: 720-26.

48. John I, D'souza JI, Jagdish K, Saboji S, Killedar G and Harinath N: Design and evaluation of benzoyl peroxide microsponges to enhance therapeutic efficacy in acne treatment. Accepted for presentation in $20^{\text {th }}$ FAPA Congress, Bangkok, Thailand 2004: 28.

49. Jain V and Singh R: Dicyclomine-loaded eudragit based microsponge with potential for colonic delivery Preparation and characterization. Tropical Journal of Pharmaceutical Research 2010; 9(1): 67-72.

50. Orlu M, Cevher E and Araman A: Design and evaluation of colon-specific drug delivery system containing Flurbiprofen microsponges. International Journal of Pharmaceutics 2006; 318: 103-17.

51. Shaheen SZ, Bolla K, Vasu $K$ and Singara CM: Antimicrobial activity of the fruit extracts of Coccinia indica. African Journal of Biotechnology 2009; 8(24): 7073-07.

52. Kawashima Y, Niwa T, Takeuchi H, Hino T and Itoh Y: Control of prolonged drug release and compression properties of ibuprofen microsponges with acrylic 
polymer, eudragit RS, by changing their intraparticle density. Chemical and Pharmaceutical Bulletin 1992; 40: 196-01.

53. Aritomi H, Yamasaki $\mathrm{Y}$ and Yamada $\mathrm{K}$, Honda $\mathrm{H}$ and Koshi M: Development of sustained release formulation of chloropheniramine maleate using powder coated microsponges prepared by dry impact blending method. Journal of Pharmaceutical Science and Technology 1996; 56: $49-56$

54. Chen G, Ushida T and Tateishi T: A Biodegradable hybrid sponge nested with collagen microsponges. Journal of Biomedical Materials and Research 2000; 51: 273-9.

55. Kanematsu A, Marui A, Yamamoto S, Ozeki M, Hirano Y and Yamamoto M: Type I collagen can function as a reservoir of basic fibroblast growth factor. Journal of Controlled Release 2004; 94: 281-92.

56. Iwai $\mathrm{S}$, Sawa $\mathrm{Y}$, Ichikawa $\mathrm{H}$, Taketani $\mathrm{S}$ and Uchimura E: Biodegradable polymer with collagen microsponge serves as a new bioengineered cardiovascular prosthesis. Journal of Thoracic and Cardiovascular Surgery 2014; 128: 472-9.

57. Chen G, Sato T, Ohgushi H, Ushida T, Tateishi $T$ and Tanaka J: Culturing of skin fibroblasts in a thin PLGAcollagen hybrid mesh. Biomaterials 2005; 26: 2559-66.

58. Iwai S, Sawa Y, Taketani S, Torikai K, Hirakawa K and Matsuda H: Novel tissue-engineered biodegradable material for reconstruction of vascular wall. Journal of Thoracic and Cardiovascular Surgery 2005; 80: 1821-7.

59. Abdelmalak NS and El-Menshawe SF: A new topical fluconazole microsponge loaded hydrogel: preparation and characterization. International Journal of Pharmacy and Pharmaceutical Sciences 2012; 4(1): 460-68.

60. Amrutiya N, Bajaj A and Madan M: Development of microsponge for topical delivery of mupirocin. American Association of Pharmaceutical Scientists 2009; 10(2): 40209.

61. Comoglu T, Gonul N and Baykara T: Preparation and invitro evaluation of modified release ketoprofen microsponges. IIFarmaco 2003; (58): 101-06.

62. Jelvehgari M, Siahi-Shadbad MR, Azarmi S, Martin G and Nokhodchi A: The microsponge delivery system of benzoyl peroxide: preparation, characterization and release studies. International Journal of Pharmaceutics 2006; 308(1-2): 124-32.

63. Martin A, Swarbrick J and Cammarrata A: In: Physical Pharmacy. Physical Chemical Principles in Pharmaceutical Sciences 1991; 3: 527.

64. Emanuele $\mathrm{AD}$ and Dinarvand R: Preparation, characterization and drug release from thermo responsive microspheres. International Journal of Pharmaceutics 1995; 118(2): 237-42.

65. Kilicarslan, $\mathrm{M}$ and Baykara, $\mathrm{T}$ : The effect of the drug/polymer ratio on the properties of verapamil $\mathrm{HCl}$ loaded microspheres. International $\mathbf{J}$ of Pharmaceutics 2003; 252: 99-109.

66. Jayaweera DM: Medicinal Plants (Indigenous and exotic) used in Ceylon. Part-2. A Publication of the Natural Sciences Council of Srilanka. Colombo 1980.

67. Martin A, Swarbrick J, Cammarata A: Physical Pharmacy. Physical chemical principles in the pharmaceutical sciences. Varghese Publication House, Edition $3^{\text {rd }}, 1991$.

68. Aloorkar NH, Kulkarni AS andIngale DJ: Microsponge as innovative drug delivery system. International Journal of Pharmaceutical Sciences and Nanotechnology 2012; 5(1).

69. Rawlins EA: Text Book of Pharmaceutics. Edition $8^{\text {th }}$, 131.

70. Barkai A, Pathak V and Benita S: Polyacrylate (Eudragit retard) microspheres for oral controlled release of nifedipine. I. Formulation design and process optimization. Drug Develop and Industrial Pharmacy 1990; 16: 2057-75.

71. Emanuele $\mathrm{AD}$ and Dinarvand R: Preparation, characterization and drug release from thermo responsive microspheres. International Journal of Biopharmaceutics 2014; 5(1): 39-46.

72. Amrutiya N, Bajaj A and Madam M: Development of microsponges for topical delivery of mupirocin. American Association of Pharmaceutical Science and Technology 2009; 10(2): 402-409.

73. Rani S, Hiremath R and Harsha N: Text book of industrial Pharmacy. 126.

74. Kumar J, Muralidharan S and Parasuraman S: Evaluation of antifungal activity of sustained release microsponge enriched fluconazole gel for penile candidiasis in male rats. International Journal of Pharmatech Research 2014; 6(6): 1888-97.

75. Kumar SP: Microsponge as the versatile tool for drug delivery. International Journal of Research in Pharmacy and Chemistry 2011; 1(2): 243-58.

76. Sharma R and Pathak K: Polymeric nanosponges as an alternative carrier for improved retention of econazole nitrate onto the skin through topical hydrogel formulation. Pharmaceutical Development and Technology 2011; 16(4): 367-76.

77. Trotta F, Cavalli R and Tumiatti W: Cyclodextrin-based nanosponges for drug delivery. Journal of Inclusion Phenomena and Macrocyclic Chemistry 2006; 56: 209-13.

78. Swaminathan S, Vavia PR and Trotta F: Formulation of betacyclodextrin based nanosponges of itraconazole. Journal of Inclusion Phenomena and Macrocyclic Chemistry 2007; 57: 89-94

79. Swaminathan S, Cavalli R and Trotta F: In-vitro release modulation and conformational stabilization of a model protein using swellable polyamidoaminenano sponges of beta-cyclodextrin. Journal of Inclusion Phenomena and Macrocyclic Chemistry 2010; 68:183-91.

80. Cavalli R, Akhter AK and Bisazza A: Nanosponge formulations as oxygen delivery systems. International Journal of Pharmaceutics 2010; 402(1-2): 254-7.

81. Torne S, Darandale S and Vavia P: Cyclodextrin-based nanosponges: effective nanocarrier for tamoxifen delivery. Pharmaceutical Develop and Technol 2012; 16(5): 380-92.

82. Ansari KA, Torne SJ and Vavia PR: Paclitaxel loaded nanosponges: In-vitro characterization and cytotoxicity study on MCF-7 cell line culture. Current Drug Delivery 2011; 8(2): 194-02.

83. Hu SH, Liu TY and Liu DM: Nano-ferrosponges for controlled drug release. Journal of Controlled Release 2007; 121(3): 181-9.

84. Benson JR and Kitagawa N: Polymeric microbeads and method of preparation. International Publication Number WO1995033553; 2003.

85. Zaki CM, Latif AR and Soliman II: In-vitro and in-vivo evaluation of hydroxyzine hydrochloride microsponges for topical delivery. American Association of Pharmceutical Science and Technology 2011; 12(3): 989-01.

86. Ansari KA, Vavia PR and Trotta F: Cyclodextrin-based nanosponges for delivery of resveratrol: in-vitro characterisation, stability, cytotoxicity and permeation study. American Association of Pharmceutical Science and Technology 2011; 12(1): 279-86.

87. Swaminathan S, Pastero L and Serpe L: Cyclodextrinbased nanosponges encapsulating camptothecin: physicochemical characterization, stability and cytotoxicity. European Journal of Pharmceutics and Biopharmaceutics 2010; 74(2): 193-01. 
88. Lee JB, Hong $\mathrm{J}$ and Bonner DK: Self-assembled RNA interference microsponges for efficient siRNA delivery. Natural Materials 2012; 11(4): 316-22.

89. Maiti S, Kaity S and Ray S: Development and evaluation of xanthan gum-facilitated ethyl cellulose microsponges for controlled percutaneous delivery of diclofenac sodium. Acta Pharmceutica 2011; 61: 257-70.

90. Afrasim M, Deb TK, Osmani AM, Bhosale RR and Umme $\mathrm{H}$ : Fabrication, characterization, and evaluation of microsponge delivery system for facilitated fungal therapy, Journal of Basic and Clinical Pharmacy 2016; 7(2): 39-48.

91. Srivastava R and Pathak K: Micosponges: a futuristic approach for oral drug delivery, Expert Opinion on Drug Delivery 2012; 9(7): 863-78.

92. Rastogi V, Shukla S, Singh R, Lal N and Yadav P: Microspheres: a promising drug carrier. Journal of Drug Delivery and Therapeutics. 2016; 6(3): 18-26. https:// doi.org/10.22270/jddt.v6i3.1196.

How to cite this article:

Sharma S, Sharma A and Kaur C: Microsponges: as a topical drug delivery system. Int J Pharm Sci \& Res 2020; 11(2): 524-34. doi: 10.13040/IJPSR.0975-8232.11(2).524-34.

All @ 2013 are reserved by International Journal of Pharmaceutical Sciences and Research. This Journal licensed under a Creative Commons Attribution-NonCommercial-ShareAlike 3.0 Unported License.

This article can be downloaded to Android OS based mobile. Scan QR Code using Code/Bar Scanner from your mobile. (Scanners are available on Google Play store) 